generation. It was also specific to certain types of tissue, such as the liver and skeletal muscle. Both generations showed reduced expression of genes that synthesize lipids in the liver. This makes them prone to developing glucose intolerance, which can lead to diabetes.

The results suggest that the epigenetic effects of maternal malnutrition affect both the offspring and the next generation.

Cell Metab. http://doi.org/sms (2014)

\section{MICROBIOLOG}

\section{Drug resistance from manure}

Dairy-cow manure that is spread on farmland could also be spreading a wide variety of genes encoding antimicrobial resistance, reports a Connecticut-based team.

Jo Handelsman at Yale University in New Haven and her team analysed DNA from five samples of manure from dairy cows (pictured) and found 80 genes from a variety of bacteria that encode proteins for resistance to a wide range of antibiotics. Among them was a new group of genes that are specific to cow manure and confer resistance to the antibiotic chloramphenicol, which is used clinically. The protein sequences deduced from the 80 genes were, on average, only $50-60 \%$ identical to known amino-acid sequences.

Cow manure is commonly used as a crop fertilizer, and this could be one way in which resistance genes disperse beyond the dairy farm, the team suggests.

mBio 5, e01017-13 (2014)

\section{PHYSICS}

\section{Heavy neutrino may be dark matter}

'Sterile' neutrinos could be the source of a mysterious signal believed to be coming from dark matter in galaxy clusters.

Standard neutrinos are thought to be too light to be candidates for dark matter. But Kevork Abazajian of the University of California, Irvine, calculated how the hypothetical sterile neutrinos, which theories suggest could have larger masses than standard neutrinos, might decay to produce X-ray emissions such as those seen earlier this year by the XMM-Newton and Chandra $\mathrm{X}$-ray space telescopes.

By factoring in the particles' interactions in the early Universe, Abazajian shows how sterile neutrinos could also be better than other darkmatter models for explaining how mass is distributed in the Milky Way.

Phys. Rev. Lett. 112, 161303 (2014)

\section{MARINE BIOLOEY}

\section{Sea life booms in hot climates}

Deep-sea ecosystems are sensitive to global temperature variations on shorter timescales than previously thought.

Moriaki Yasahara at the University of Hong Kong and his colleagues took

samples of a fossil-rich sediment core from the northeastern Atlantic Ocean to study variability in the abundance of deepwater species over the past 20,000 years. Focusing on a scale of decades to centuries, the researchers found that abrupt glaciations such as the Younger Dryas cooling period some 13,000 years ago led to significant increases

SOCIAL SELECTION Popmanaraicas

\title{
ENCODE debate revived online
}

In the social-media age, scientific disagreements can quickly become public - and vitriolic. A report from the ENCODE (Encyclopedia of DNA Elements) Project consortium proposes a framework for quantifying the functional parts of the human genome. It follows a controversial 2012 Nature paper by the same group that concluded that $80 \%$ of the genome is biochemically functional (Nature 489, 57-74; 2012). Dan Graur, who studies molecular evolutionary bioinformatics at the University of Houston in Texas and is a vocal ENCODE critic, weighed in on this latest report. ENCODE's "stupid claims" from 2012 have finally come to back to "bite them in the proverbial junk", Graur wrote on his blog. The targets noticed. "Some people seek attention through hyperbole and mockery," says the report's first author Manolis Kellis, a computer scientist at the Massachusetts Institute of Technology in Cambridge. "We should stay focused on the issues."

Proc. Natl Acad. Sci. USA 111, 6131-6138 (2014)

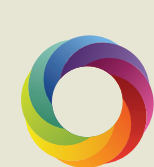
Based on data from altmetric.com. Altmetric is supported by Macmillan Science and Education, which owns Nature Publishing Group.

ONATURE.COM

For more on popular papers: go.nature.com/9hsjcw in biodiversity on the ocean floor within around 100 years. Weakening ocean-circulation patterns during these surfacecooling events resulted in warmer deep-ocean waters.

This high sensitivity to rapid temperature variation shows how marine biodiversity could respond suddenly to humaninduced climate change this century, the authors say. Global Ecol. Biogeogr. http://doi. org/sk7 (2014)

\section{AGRICULTURE \\ Maize vulnerable to drought}

Yields of maize (corn) have increased in the central United States since 1995, but so has the crop's sensitivity to drought.

David Lobell from Stanford University in California and his colleagues studied yields of US maize (pictured) and daily weather data for between 1995 and 2012, focusing on a measure of atmospheric dryness called vapourpressure deficit. The authors

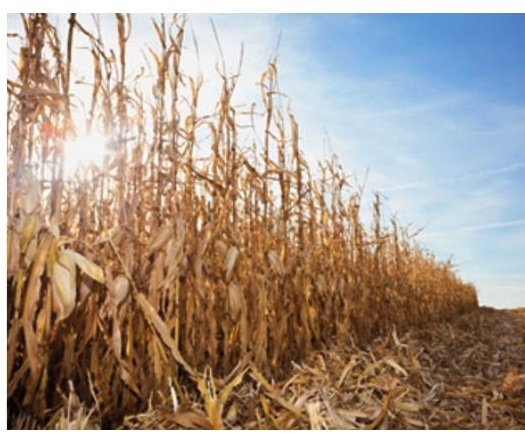

found that yields have been increasing overall, but in years of high vapour-pressure deficits, maize grew more slowly. The team says that the practice of packing plants close together could be boosting this sensitivity.

Climate change could have larger negative effects on US maize crops than previously thought, because vapourpressure deficit in the region is predicted to rise as the climate warms, the authors say.

Science 344, 516-519 (2014)

\section{$\rightarrow$ NATURE,COM}

For the latest research published by Naturevisit:

www.nature.com/latestresearch 